\title{
ITRACONAZOL NO TRATAMENTO DA PITIRÍASE VERSICOLOR: COMPARAÇÃO ENTRE 5 E 7 DIAS DE TRATAMENTO
}

\author{
Luiz Carlos CUCÉ (1), Walter BELDA JR. (2) \& Eliane Barbosa de Oliveira RIBEIRO (3)
}

\begin{abstract}
RESUMO
Os autores analisam os resultados obtidos no tratamento da pitiríase versicolor com o Itraconazol em estudo aberto, comparativo, na dose de $200 \mathrm{mg} /$ dia por 5 e 7 dias.

A diagnose foi estabelecida pelo exame clínico, fluorescência pela luz de Wood exame micológico direto, sendo os pacientes reavaliados no 21: e 35: dia após o início da terapêutica.

Ambos os esquemas de tratamento utilizados mostraram-se eficazes. Os índices de cura clínica e micológica, obtidos no último controle, foram de $90 \%$ para o grupo tratado por 5 dias e $95 \%$ para o grupo tratado por 7 dias. A tolerância à droga foi boa, tendo sido registrados efeitos colaterais em dois pacientes do grupo tratado por 7 dias e em um paciente do grupo tratado por 5 dias. Como não houve diferença estatisticamente significante entre os grupos tratados, os autores recomendam o esquema de tratamento mais curto, ou seja, $200 \mathrm{mg} /$ dia por 5 dias.
\end{abstract}

UNITERMOS: Pitiriase versicolor; Itraconazol; Tratamento.

\section{INTRODUÇÃo}

Vários tratamentos tópicos são conhecidos e sabidamente eficazes na maioria dos casos de pitiriase versicolor. A terapêutica sistêmica desta dermatose, tão freqüente em nosso meio, foi iniciada com o Cetoconazol ${ }^{2,4}$, 12, 14, 15, sendo que a administração da dose diária de $200 \mathrm{mg}$, por período de 10 ou 20 dias, revelou resultados semelhantes ${ }^{13}$. Entretanto, formas extensas e recidivantes necessitam eventualmente de tratamentos repetidos.
Atualmente, o Itraconazol, novo derivado imidazólico, tem sido usado com sucesso na terapêutica de micoses superficiais e profundas, in clusive na própria pitiríase versicolor ${ }^{1,3}, 5,6,7$. 8. $10,11,16$, onde seu mecanismo de ação parece se efetuar sobre o citocromo P-450, levando a um distúrbio na biossíntese de esteróis da parede celular dos fungos, com conseqüente morte de parasitas ${ }^{9}$.

HEINS-VACCARI ${ }^{9}$ compara sua açāo in vi-

Trabaho realizadic na Divisāo de Dermatologia do Hospital das Clínicas da Faculdade de Medicina da Universidade de Sảo Paulo (Serviço do Prof. Sebastião A. P. Sampaio), Av. Dr. Enéas de Carvalho Aguiar, 255 - 05403 - Sāo Paulo, SP, Brasil.

(1) Professor Associado do Departamento de Dermatologia da Faculdade de Medicina da Universidade de São Paulo. São Paulo, SP, Brasil.

(2) Auxiliar de Ensino do Departamento de Dermatologia da Faculdade de Medicina da Universidade de Sāo Paulo. Sāo Paulo, SP, Brasil.

(3) Residente de Dermatologia do Hospital das Clínicas da Faculdade de Medicina da Universidade de São Paulo. São Paulo, SP, Brasil.

Endereço para correspondència: Prof. Luiz Carlos Cucé - Departamento de Dermatologia da FMUSP - IC HC - Av. Dr. Enéas de Carvalho Aguiar, $255-3$ : andar - São Paulo, SP, Brasil. 
CUCÉ, L. C.; BELDA Jr., W. \& RIBEIRO, E. B. de O. - Itraconazol no tratamento da pitiriase versicolor: comparaçào entre 5 e 7 dias de tratamento. Rev. Inst. Med. trop. S. Paulo, 32(3): 181-184, 1990.

tro sobre a Malassezia furfur com os resultados obtidos com o Cetoconazol e BAY n: 7133 , concluindo ser a atuação do Cetoconazol in vitro superior às outras duas drogas.

Assim sendo, os autores se propuseram a estudar esta nova droga na pitiríase versicolor, em 2 esquemas terapêuticos: $200 \mathrm{mg}$ ao dia, durante 5 e 7 dias.

\section{PACIENTES E MÉTODOS}

Foram estudados 42 pacientes de ambos os sexos, com idade superior a 17 anos, divididos em 2 grupos de 21 pacientes cada. Não foram incluídos no estudo pacientes grávidas ou lactantes; pacientes com antecedentes de afecçāo hepática; pacientes em uso de antimicótico tópico que não tivesse sido suspenso pelo menos 3 dias antes da inclusão no estudo ou antimicótico oral que nāo tivesse sido suspenso pelo menos 10 dias antes do início do tratamento.

A diagnose foi estabelecida pelo exame clínico,fluorescência pela luz de Wood e exame micológico direto.

Os dois grupos de tratamento mostraram-se estatisticamente homogêneos com relaçāo ao sexo (Teste de Fisher $-\mathrm{p}=0,500$ n.s.), idade (Teste de $\mathrm{t}$-Student $-\mathrm{t}=1.903$ n.s.) e características da doença (Teste de Fisher $-\mathrm{p}=0,367$ n.s. e Teste de $t$-Student $-t=1,332$ n.s.) dos 21 pacientes do grupo tratado por 5 dias, 15 eram do sexo masculino e 6 do sexo feminino, com idade variando entre 18 e 52 anos, média de 31,5 anos. Destes pacientes, 16 não haviam feito tratamento anterior e 5 apresentavam recidivas de tratamentos prévios. O tempo de evoluçāo da afecção variou de 20 dias à 1 ano.

No grupo de pacientes tratados por 7 dias, 14 eram do sexo masculino e 7 do sexo feminino, com idade variando de 18 a 45 anos, média de 26,5 anos. Destes pacientes, 11 não haviam fejto tratamento anterior e 10 apresentavam recidivas após terapêtica tópica. O tempo de evolução variou de 10 dias à 5 anos.

Em ambos os grupos as lesōes se apresen tavam de forma localizada, sendo que apenas 1 caso em cada grupo apresentava lesōes disse minadas.
O Itraconazol foi administrado por via oral, dividido em 2 tomadas diárias, após as refeiçōes, na dose total diária de $200 \mathrm{mg}$, por 5 ou 7 dias, conforme o grupo estudado.

Foram realizados controles clínicos e laboratoriais na consulta de pré-tratamento, no $21^{\circ}$ e 35. dia após o início da terapêtica. A comparação dos resultados obtidos nos exames micológicos, en tre os grupos de 5 e 7 dias de tratamento, foi realizado através do teste exato de Fisher. Também foi registrado a eventual ocorrência de efeitos colaterais.

\section{RESULTADOS}

A avaliaçāo clínica realizada no pré-tratamento revelou que todos os pacientes apresentavam descamação ao iniciar a terapêtica. No 1. controle (21 dias após o início do tratamento) a descamação havia desaparecido em quinze pacientes de cada grupo e permanecido em grau leve em seis pacientes. No 2 : controle (35 dias após o início do tratamento) apenas dois pacientes de cada grupo ainda apresentavam descamação de grau leve. Portanto, a evolução deste sinal clínico foi idêntica para ambos os grupos de tratamento, nāo havendo diferença estatísticamente entre os grupos em nenhum período de avaliação (Teste de Mann-Whitney)

A comparação da intensidade da descamação, nos dois controles, com o pré-tratamento, foi realizada pelo Teste de Friedman com contrastes, encontrando-se significância estatística ( $p<0,001)$ para a diminuição da intensidade deste sinal.

Quanto ao prurido, estava presente em ape nas 8 pacientes ao inicio da terapêtica, tendo desaparecido em todos esses pacientes, já no 1 . controle.

A Tabela 1 mostra os resultados obtidos do exame direto e đa fluorescência pela lâmpada de Wood.

No grupo tratado por 5 dias, a avaliação realizada no 21: dia revelou cura clínica e micológica em 18 pacientes e persistentes áreas descamativas, com pesquisa micológica direta positiva e fluorescência específica ao exame pela luz de Wood em 3 pacientes. 
CUCE, L. C.; BELDA Jr., W. \& RIBEIRO, E. B. de O. - Itraconazol no tratamento da pitiriase versicolor: comparaçăo entre 5 e 7 dias de tratamento. Rev. Inst. Med. trop. S. Paulo, 32(3): 181-184, 1990.

\section{RESULTADOS DOS EXAMES MICOLÓGICOS}

\begin{tabular}{|c|c|c|c|c|c|c|c|}
\hline & & \multicolumn{3}{|c|}{ Grupo 5 dias de tratamento } & \multicolumn{3}{|c|}{ Grupo 7 dias de trabamento } \\
\hline & & $\begin{array}{c}\text { Pré- } \\
\text { tratamento }\end{array}$ & $\begin{array}{l}21 \text { dias } \\
\text { após inicio } \\
\text { tratamento }\end{array}$ & $\begin{array}{c}35 \text { dias } \\
\text { após inicio } \\
\text { tratamento }\end{array}$ & $\begin{array}{c}\text { Pré } \\
\text { tratamento }\end{array}$ & $\begin{array}{l}21 \text { dias } \\
\text { após início } \\
\text { tratamento }\end{array}$ & $\begin{array}{c}35 \text { dias } \\
\text { após inicio } \\
\text { tratamento }\end{array}$ \\
\hline \multirow{2}{*}{ Exame direto } & Negativo & - & $18(86 \%)$ & $19(90 \%)$ & - & $19(90 \%)$ & $20(95 \%)$ \\
\hline & Positivo & 21 & $3(14 \%)$ & $2(10 \%)$ & 21 & $2(10 \%)$ & $1\{5 \%\}$ \\
\hline \multirow{2}{*}{ Luz de Wood } & Negativo & - & $18(86 \%)$ & $19(90 \%)$ & - & $19(90 \%)$ & $20(95 \%)$ \\
\hline & Positivo & 21 & $3(14 \%)$ & $2(10 \%)$ & 21 & $2 \cdot(10 \%)$ & $1(5 \%)$ \\
\hline
\end{tabular}

Comparaçāo entre grupos de tratamento: Nāo houve diferença estatisticamente significante entre os grupos em nenhum período de avaliaçāo (Teste exato de Fisher).

No con trole realizado no $35^{\circ}$ dia após o início do tratamento, a cura clínica e laboratorial foi obtida em 19 pacientes $(90 \%)$, permanecendo a micose em atividade em 2 pacientes. Neste grupo de tratamento apenas um paciente referiu efeito colateral, ou seja, prurido nas mãos e pés, de grau moderado, que regrediu ao término da terapêutica.

Quanto ao grupo tratado por 7 dias, o controle realizado no 21 . dia revelou cura clínica e micológica em 19 pacientes e exame direto positivo, acompanhado de fluorescência à luz de Wood, em 2 pacientes. Na avaliação realizada no $35^{\circ}$ dia, 20 pacientes $(95 \%)$ apresentaram-se clínica e micologicamente curados, persistindo a positividade ao exame direto e à luz de Wood, em apenas um paciente. Os efeitos colaterais registrados neste grupo resumiram-se a pirose, de grau leve, observada em apenas 2 pacientes e regredindo ao término do tratamento. A comparaçāo dos resultados obtidos nos exames micológicos, entre os grupos de 5 e 7 dias de tratamento, foi realizada através do Teste Exato de Fisher, demonstrando não haver diferença estatisticamente significante entre os grupos, em nenhum período de avaliaçāo.

\section{COMENTÁRIOS E CONCLUSŌES}

A análise dos resultados obtidos no tratamento da pitiriase versicolor como Itraconazol, na dose de $200 \mathrm{mg} / \mathrm{dia}$, em esquemas de 5 e 7 dias de duração, revelou, no último controle, ín dices de eficácia semelhantes para os dois gru- pos, ou seja, de 90 e $95 \%$ respectivamente. A tolerância à droga foi boa, sendo que os poucos efeitos colaterais registrados foram transitórios, de pequena intensidade, não demandando a interrupção do tratamento. Os dados obtidos estāo em concordância com os relatados na literatura e indicam que o Itraconazol desponta como uma droga realmente eficaz na terapêutica da pitiríase versicolor, uma vez que estes índices obtidos foram semelhantes aos obtidos com o uso de Cetoconazol na dose de $200 \mathrm{mg}$ por $30 \mathrm{dias}^{4}$, porém com esquema terapêutico de somente 5 e 7 dias, e sem recidivas posteriores em nenhum dos dois grupos.

Uma vez que os índices de cura obtidos em ambos os esquemas do tratamento não revelaram diferença estatisticamente significante, deve-se optar quando da utilização desta droga, pelo esquema de menor duração, ou seja, 200 $\mathrm{mg} /$ dia, durante 5 dias.

\section{SUMMARY}

\section{Itraconazole in the treatment of pityriasis versicolor:a comparison between 5 and 7 days of treatment}

The authors present the results obtained in the treatment of pityriasis versicolor with Itraconazole, comparing two schemes of treatment, in an open trial: $200 \mathrm{mg}$ daily, for 5 and 7 days. The clinical diagnosis was confirmed by Wood's light and mycologically, by direct examination. 
CUCÉ, L. C.; BELDA Jr., W. \& RIBEIRO, E. B. de O. - Itraconazol no tratamento da pitiriase versicolor: comparaçāo entre 5 e 7 dias de tratamento. Rev. Inst. Med. trop. S. Paulo, 32(3): 181-184, 1990.

The patients were controlled 21 and 35 days after beginning the treatment.

The evaluation of the results showed that both schemes were effective.

At the second control, there was clinical and mycologic cure in $90 \%$ of the patients from the 5-day group and in $95 \%$ of the patients from the 7-day group.

The tolerance was good: 2 patients from the 7-day group and 1 patient from the 5-day group showed side-effects. Since there was no significant difference between the two schemes of treatment, the authors recommend the shortest one, that is, $200 \mathrm{mg}$ daily for 5 days.

\section{REFERÊNCIAS BIBLIOGRÁFICAS}

1. BIGgio, P.; ASTE, N. \& PAU. M. - Primi resultati dell imprego dell'itraconazolo (51211) nel trattamento della pitiriasis versicolor. Derm. Clin., 3: 251-255, 1986.

2. BORELLI, D.; RODRIGUES, H. \& MARGANO, C. - Pity riasis versicolor: tratamento "per os" con Ketoconazole. Rev. Fund. José María Vargas (Caracas), 3(10): 19-23, 1979.

3. CAUWENBERGH, G. \& DE DONCKER, P. - Itraconazole $(R-51211)$ : a clinical review of its antimycotic activity in dermatology, gynecology and internai medicine. Drug. Develop. Res., 8: 317-323, 1986.

4. CUCÉ, L. C.; SAlebian, A. \& SAMPAiO, S. A. P. - Tra tamento sistêmico das micoses superficiais pelo Ketoconazole. An. bras. Derm., 57: 5-7, 1982.

5. DEL PALACIO HERMANZ, A.; FRIAS INTESTA, J.; GONZALEZ VALLE. O.; BORGES. M.; VAN CUTSEN. J. \& CAUWENBERGH, G. - Itraconazole (R-51211) therapy in pityriasis versicolor. Brit. J. Derm., 115: 212-225, 1986.
6. DEL PALACIO HERMANZ, A.; VICENTE, S. D.; RAMOS, F. M. \& BELAUSTEGUI, A. R. N. - Randomized comparative clinical trial of itraconazole and selenium sulfide shampoo for the treatment of pityriasis versicolor. Rev. infect. Dis., 9 (supp. 1): S121-S127, 1987.

7. ESTRADA, R.A. - Itraconazole in pityriasis versicolor. Rev. infect. Dis., 9 (supp. 1): S128-S130, 1987.

8. GALIMBERTI, R. L.; VILLALBA, I; GALARZA, S.; RAI MUNDI, A. \& FLORES, V. - Itraconazole in pityriasis versicolor: ultrastructural changes in Malassezia furfur produced during treatment. Rev. infect. Dis., 9 (supp. 1): S134-S138, 1987.

9. HEINS-VACCARI, E. M.; MELO, N. T.; LACAZ, C. S.; DEL NEGRO, G. \& SALEBIAN, A. - Açáo in vitro do itraconazol ( $R-51211$ ) sobre a Malassezia furfur. An. bras. Derm., 62: 75-78, 1987.

10. MORALES-DORIA, M. - Pityriasis versicolor: efficacy of two five-day regimens of itraconazole. Rev. infect. Dis., 9 (supp. 1): S131-S133, 1987.

11. PANCUNESI, E. \& DIFONZO, E. - Treatment of derma tophytosis and pityriasis versicolor with itraconazole. Rev. infect. Dis., 9 (suppl. 1): S109-S113, 1987.

12. SAMPAIO, S. A. P.; FREITAS, T. H. P. \& SAGOBAL, M. F. - Tratamento de pitiríase versicolor pelo Ketocona zole. An. bras. Derm., 58: 245-248, 1983.

13. SAMPAiO, S. A. P.; ZEMINIAN, P. S. \& SALEBIAN, A. - Ketoconazole na pitiriase versicolor: estudo comparativo entre dois períodos de tratamento, 10 e 20 dias. An. bras. Derm., 60: 41-44, 1985.

14. SAVIN, R. C. - Systemic Ketoconazole in tinea versico lor: a double-blind evaluation and 1-year follow-up. J. Amer. Acad. Derm., 10: 824-830, 1984.

15. URCUYO, F. G. \& ZAIAS, N. - The successfuI treatment of pityriasis versicolor by sistemic Ketoconazole. J. Amer, Acad. Derm., 6: 24-25, 1982.

16. ZEMINIAN, P. S.; BENDER, A. T.; RIBEIRO, E. B. O. SALEBIAN, A. \& SAMPAIO, S. A. P. - Tratamento sistémico da pitiriase versicolor pelo itraconazol. An. bras. Derm., 62: 187.188, 1987.

Recebido para publicação em 01/09/1989. 